\title{
Engineering networks simulation and assessment of the mathematical model accuracy
}

\author{
Umarkhodzha Baxramov", Uchqun Umarov, and Axror Obidzhonov \\ Tashkent State Transport University, Tashkent, Uzbekistan
}

\begin{abstract}
The article shows the way to implement a quasilinear mathematical model of flow distribution in pipeline engineering networks that is effective in a wide range of changes in the multidimensional random vector of loads at network nodes and provides reliable determination of the parameters of the probability distribution functions of flows in active and passive network elements. The proposed model consists of determining the matrix of generalized network parameters-the load distribution coefficients along the branches of the circuit, calculated at the point corresponding to the mathematical expectation of the node loads. Based on the obtained model, the convergence of the results obtained with the results of simulation of engineering networks is proved using a numerical experiment on an electronic computer. The effectiveness of the developed model, the corresponding algorithms and a set of programs for an electronic computer is shown - the value of the criterion of reduced costs for parametric optimization of engineering networks can be reduced by 5 $7 \%$ compared to the methods used in practice. The possibility of obtaining at the design stage the equivalent hydraulic characteristics of engineering networks in the form corresponding to the data of experimental measurements of pressures at the nodes of real complex engineering networks is proved.
\end{abstract}

\section{Introduction}

The main task of mathematical modelling of engineering networks in this work is to assess the reliability of the mathematical model of stochastic flow distribution proposed in $[1,2]$. For simulation, three calculation schemes of the utility network are used, shown in Figures 1,2 and 3. It is easy to see that these calculation schemes differ in their dimensionality, the number of nodes and branches, which allows us to objectively reveal the advantages of the mathematical model on networks of varying complexity.

\section{Materials and Methods}

\footnotetext{
*Corresponding author: umarxodja@bk.ru
} 
Simulation is reduced to carrying out a large number of calculations of the steady-state flow distribution at various values of the loads in the nodes of the network circuit. With the accumulation of data from such calculations, it becomes possible to estimate the parameters of the following probability distribution functions:

1. Flow values in each passive circuit element (network section) qi.

2. The values of the head loss in each passive element - hi.

3 . The values of the total feeds of the target product to all nodes of the scheme - $\Sigma$ Qi.

4. The pressure difference values at the active sources and at the dictating point of the circuit - $\mathrm{H} \Delta$ (these values correspond to the largest values in the matrix [1]).

To simplify the analysis, a normal distribution of all random variables is assumed. Therefore, only two non-random parameters are determined for each of the distributions the mathematical expectation and variance. Also, for active sources, the covariance values of the dependent random variables are determined - h0 and $\Sigma \mathrm{Qj}$, which, as shown below, is necessary to calculate the total energy consumption for the transportation of the target product.

A general algorithm for simulation (figure - 4) consists of three blocks - A1 in which random load values are generated for all consumption nodes of the target product; A2 providing the calculation of the steady-state flow distribution, and A3, designed for statistical processing of the results obtained.

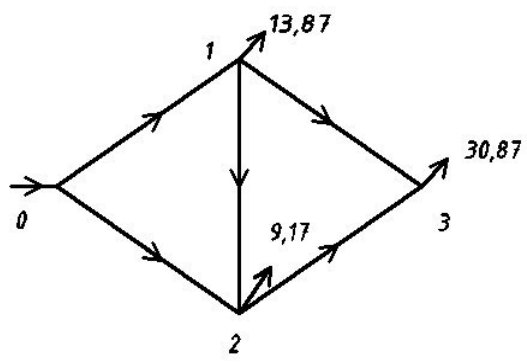

Fig. 1. The first design scheme of a utility network with 2 rings

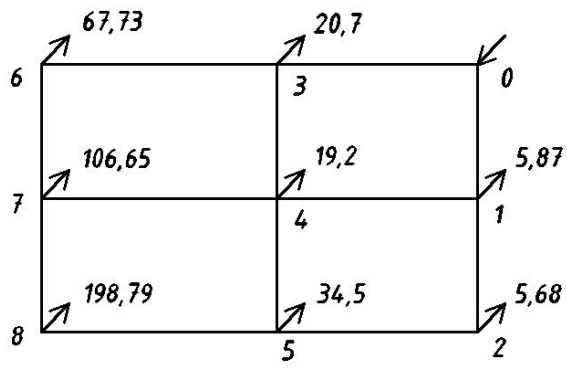

Fig. 2. The second design scheme of the utility network with 4 rings

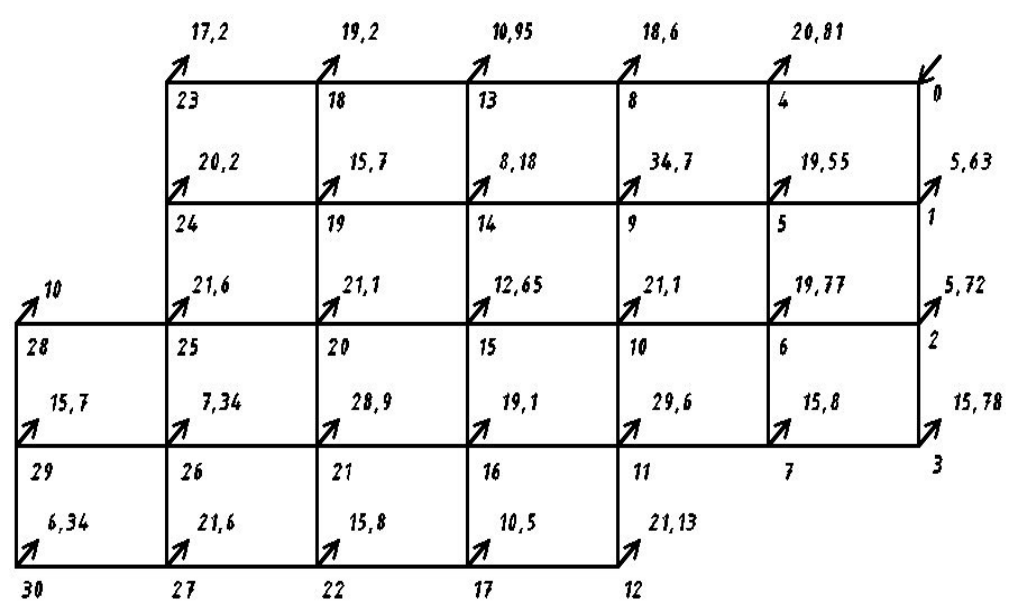

Fig. 3. The third design scheme of the utility network with 20 rings 
Block A1 built according to section data 2.1 [2]. Since the parameters of this model, in the general case, change every day of operation of the utility network, for the operation of the block, the initial information contains not only the values of the mathematical expectations of the amplitude and phase shift for each of the two harmonies but also the values of the coefficients of their variation. Also, the mathematical expectation and the coefficient of variation are also characterized by the value in $(2,3)$ [2]. Such a volume of initial information makes it possible to fairly reliably simulate the process of consumption of the target product at any node of the calculation scheme. In this work, the process of consuming a target product in water supply systems is modelled, based on the results, the study of which is used for the initial information necessary for modelling. In the block A1, a sensor of pseudo-random numbers distributed according to the normal law with zero mathematical expectation and unit variance is provided [6].

Considering the block A2, it should be noted that almost any of the known algorithms and programs for calculating the steady-state flow distribution can be used here $[7,8]$. The only requirement for them from the standpoint of the features of simulation is the need for a fairly convenient software replacement of the values of nodal loads based on the results of the block A1. The block algorithm used in this work A2 detailed in [2] section 3.3

Block A3 the simulation algorithm is quite simple, and its essence boils down to the fact that for all elements of the network design scheme, including active elements, mathematical expectations are calculated, variance, standard deviations and coefficients of variation for each of the distributions of interest of random variables (P) by well-known [6] formulas:

$$
\begin{gathered}
M(P)=\frac{\sum P}{N} ; \quad D(P)=\frac{\sum P^{2}}{N}-[M(P)]^{2} ; \\
\sigma(P)=\sqrt{D(P) ;} \quad v_{p}=\frac{\sigma(P)}{M(P)} ;
\end{gathered}
$$

Initial information used to operate the unit A1 is presented below. The algorithm for simulation modelling of the utility network Figure 3, described in [9], is given in Table 3.1. When modelling a network, Figure 1 used data for nodes 1, 2, 3 from table 3.1, but for the network Figure 2 received data corresponding to nodes 19 from table 1 . In table 3.1, amplitude values harmonics and standard deviation are given in relative units - in shares for each of the nodes of the design scheme.

\section{Results and Discussion}

The results of the simulation modelling of three engineering networks are presented in tables $3.2 \div 3.4$. Figure 4 shows the relationship between the coefficients of variation of flows in the lines of networks $\left(v_{q}\right)$ and head losses $\left(v_{h}\right)$, obtained from modelling. Also shown here is the line corresponding to the above [1]) the relationship between these coefficients. Good agreement between the experimental and theoretical data confirms the correctness of the latter and the possibility of calculating the parameters of the distribution functions of the pressure delivery in passive elements from the data on the parameters of the flow distribution functions. 


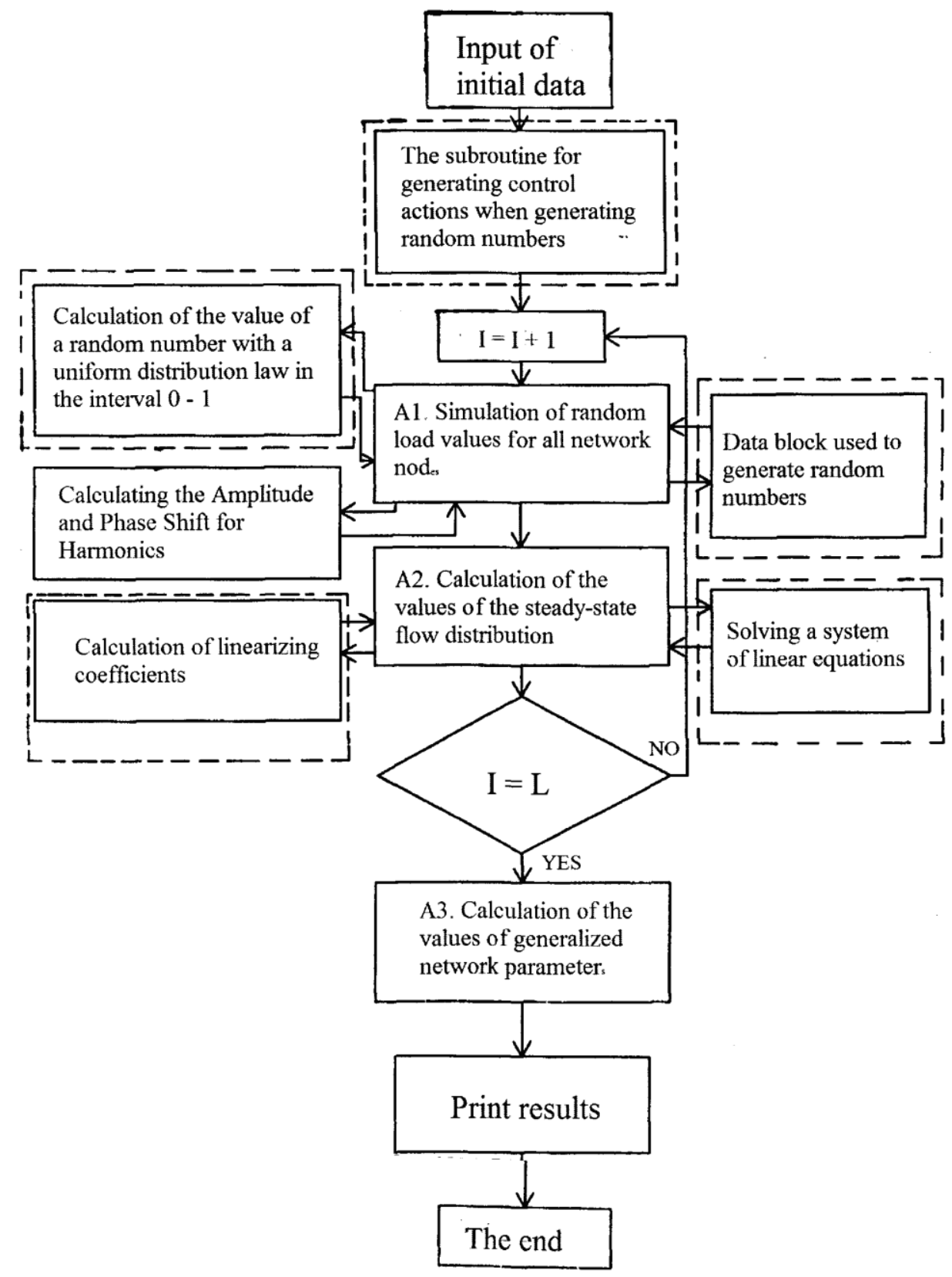

Fig. 4. Block diagram of the simulation algorithm 


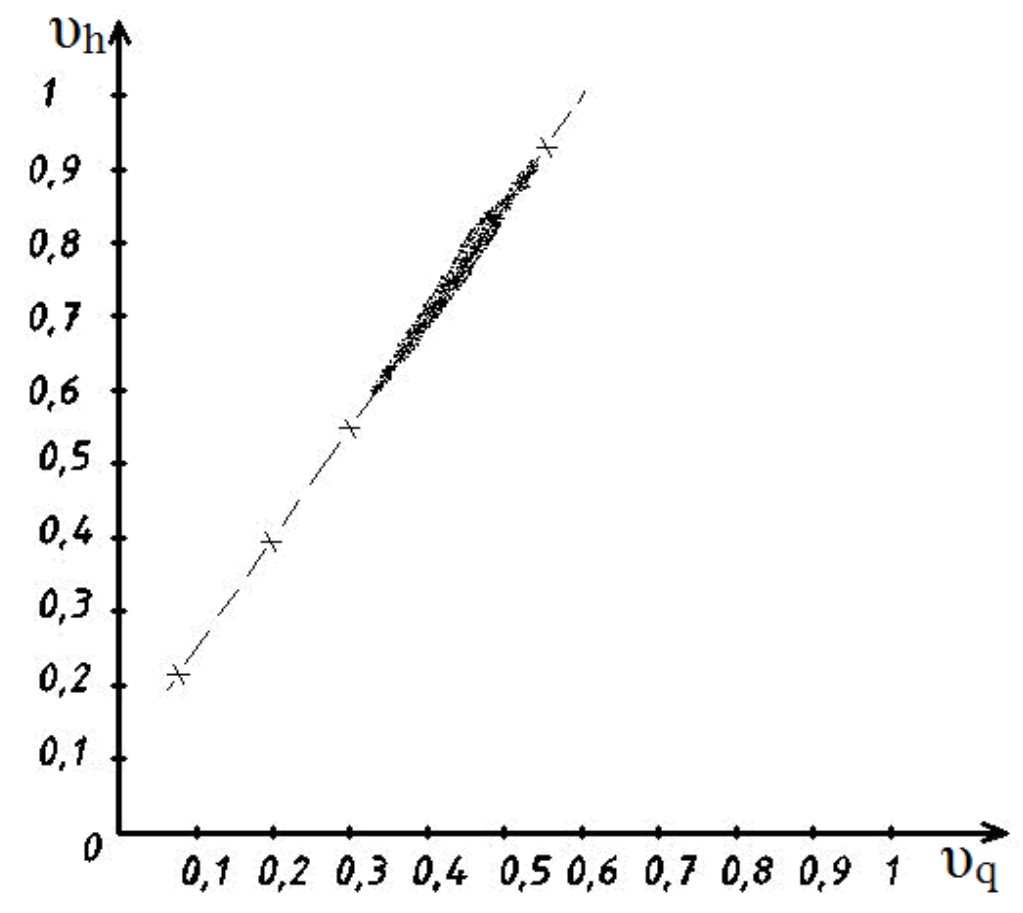

Fig.-5. $v_{q}-v_{h}$ calculation line according to (3.30) [2]

Comparison of the parameters of the distribution functions of flows in passive elements obtained during simulation and calculation by [1] and [2] (see table $2 \div 4$ ), shows that the proposed mathematical model of stochastic flow distribution in nonlinear pipeline networks provides an accuracy sufficient for practical purposes - calculation error ${ }^{q_{i}}$ less than $-8 \%$, and for $v_{q_{i}}-10 \%$.

When calculating the parameters of the distribution function of the total loads in the network ( 1 ) and head losses in the network (1) by formulas [1] and [2], [1] a single value of the correlation coefficient between the process of consumption of the target product in the nodes of the utility network was adopted $r_{i j}=0,25$. The quantity ${ }_{i j}$ obtained from the graph of figure 3.5, where the change in the variance of the total network load is shown depending on the value ${ }^{r_{i j}}$ in [1]. For all three considered networks, the value ${ }^{r_{i j}}$ at which the calculated value of the variance of the total load (in simulation modelling) coincides with the value obtained from [1]) approximately equals 0.25 . The same meaning $r_{i j}$ is also used in calculating the dispersion of head losses in the network, which is quite acceptable since the discrepancy between the simulation data and the calculation by the mathematical model of the stochastic flow distribution does not exceed $10 \%$ (see tables 2 - 4).

Calculation of the parameters of stochastic flow distribution for networks figures 3, 2 and 3, 3 very cumbersome due to the large dimension of the matrix of load distribution coefficients $C_{i j}$ and are performed only using an electronic computer.

According to the results of the calculation (network 3.3) on the graph (figure 3.6), the field of possible changes in the pressure losses in the network was constructed by $H_{\Delta}$, and total load $\sum Q_{j}$, two points of which (A and B) correspond to the limiting (smallest and largest) values of the head losses in the network at the minimum and maximum values of the total network load. 
For the correct selection of pumping equipment and tormented points, it is necessary to find the limits of the possible change in head losses in the network at different values of the total load $\sum Q_{j}$. This can be done by considering a system of two random variables $H_{\Delta}$ and $\sum Q_{j}$ assuming for each of them the normal probability distribution law. Let's consider the correlation coefficient between the values of these random variables as known, for example, take it equally as before 0.25 . We can find the so-called conditional distribution $H_{\Delta}$, that is, the laws of its distribution for various fixed values $\sum Q_{j}$, known $[6,9,10]$, that the density of the conditional distribution of two correlation normally distributed random variables is determined by the expression:

$$
\begin{gathered}
\rho\left(H_{\Delta}, \Sigma Q_{j}=\mathrm{E}\right)=\frac{1}{2 \pi \sigma\left(H_{\Delta}\right) \sigma\left(\sum Q_{j}\right) \sqrt{1-r^{2}}} \exp \\
\frac{1}{2}\left[\frac{\left(H_{\Delta}-\overline{H_{\Delta}}\right)^{2}}{\sigma^{2}\left(H_{\Delta}\right)}-\frac{2 r\left(H_{\Delta}-\overline{H_{\Delta}}\right)^{2}(E-\underline{E})^{2}}{\sigma\left(H_{\Delta}\right) \sigma\left(\Sigma Q_{j}\right)}+\frac{(E-\bar{E})^{2}}{\sigma^{2}\left(\Sigma Q_{j}\right)}\right]
\end{gathered}
$$
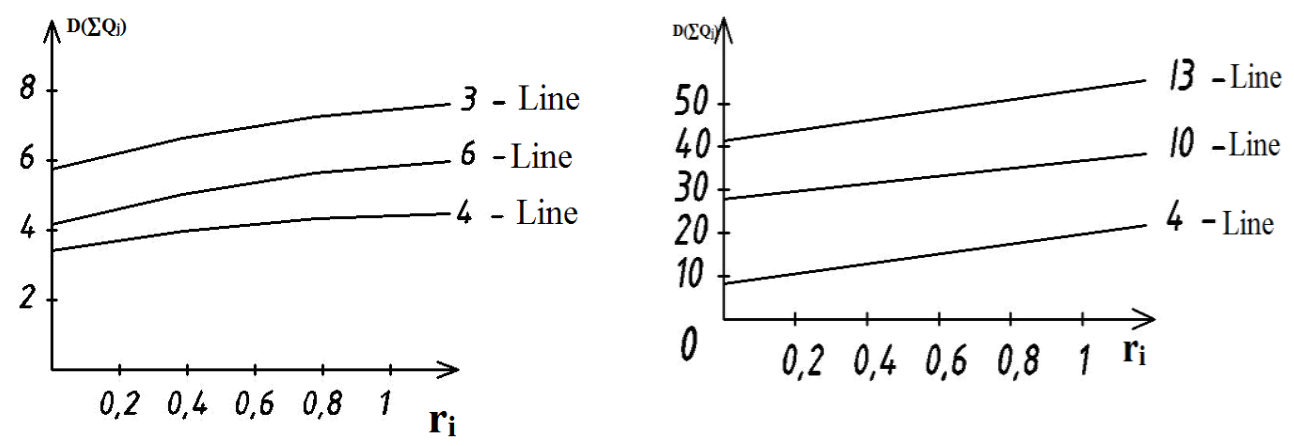

Fig. 6. Change in variance of total load network depending on the value of the coefficient correlations between the target consumption process product at network nodes. $a$ is the network in Figure $1 ; \mathrm{b}$ is the network in Figure 2. $D\left(\sum Q_{j}\right)$ is variance value total loads obtained by imitation modelling.

From (2), the probability of the appearance of different values $H_{\Delta}$ at $\sum Q_{j}=E$. In (2), all the necessary quantities are known from the results of the above-described calculation of the stochastic flow distribution. The correlation coefficient between и саn be refined based on the simulation results. For the network figure 2, the graph of values $H_{\Delta}$ for various $\sum Q_{j}$ shown in Figure 5 - the correlation coefficient here is 0.3 , which is quite close to the one used earlier. Results of calculating conditional probability distribution functions $H_{\Delta}$ at 6 values $\sum Q_{j}$ shown in figure 3.8, and the parameters of these functions are given in table 3.5 , the data of which show that the calculation for 2 quite well converges with simulation modelling and is quite consistent with the data of field experiments in engineering networks shown in figure 7. 


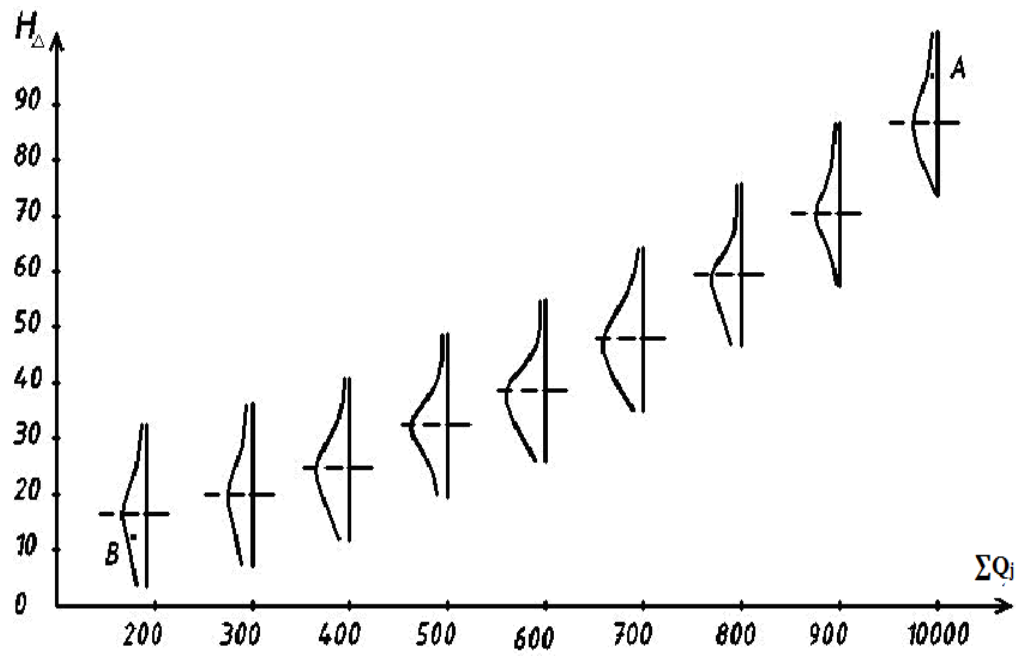

Fig. 7. Distribution functions of possible changes in head losses in the network: A - the highest value of head loss; B - the smallest value of the head loss

Comparison of simulation results and calculations of the mathematical model of stochastic flow distribution for the network in Figure 1

\begin{tabular}{|c|c|c|c|c|c|c|c|c|}
\hline \multirow{2}{*}{$\begin{array}{c}\text { Rooms } \\
\text { plot } \\
\text { networks }\end{array}$} & \multicolumn{5}{|c|}{ Simulation modelling } & \multicolumn{4}{c|}{ Math modelling } \\
\cline { 2 - 10 } & $\overline{q_{i}}$ & $v_{q}$ & $\overline{h_{i}}$ & $\overline{q_{i}}$ & $v_{q}$ & $\overline{h_{i}}$ & $\overline{q_{i}}$ & $v_{q,}$ \\
\hline 1 & 27.05 & 0.217 & 766 & 0.411 & 27.27 & 0.259 & 791.18 & 0.416 \\
\hline 2 & 36.86 & 0.215 & 755 & 0.403 & 26.64 & 0.265 & 759.15 & 0.499 \\
\hline 3 & 3.08 & 0.782 & 15.3 & 1.37 & 3.19 & 0.980 & 19.9 & 1.22 \\
\hline 4 & 15.29 & 0.258 & 249 & 0.484 & 15.12 & 0.262 & 244.4 & 0.498 \\
\hline 5 & 15.29 & 0.264 & 260 & 0.508 & $15 / 57$ & 0.256 & 258.3 & 0.487 \\
\hline $\begin{array}{c}\text { A source } \\
\text { (Node 0) }\end{array}$ & $\overline{\sum Q_{j}}=$ & $v_{\sum Q_{i}}=$ & $\overline{H_{\Delta}}=$ & $v_{H_{\Delta}}=$ & $\bar{\sum} Q_{j}$ & $v_{\sum Q_{i}}=$ & $\overline{H_{\Delta}}$ & $v_{H_{\Delta}}$ \\
& 513,56 & 0,205 & 1,4 & 0,410 & 513,1 & 0,189 & 1,35 & 0,408 \\
\hline
\end{tabular}

Comparison of simulation results and calculations of the mathematical model of stochastic flow distribution for the network in Figure 2

\begin{tabular}{|c|c|c|c|c|c|c|c|c|}
\hline \multirow{2}{*}{$\begin{array}{c}\text { Rooms } \\
\text { plot } \\
\text { network } \\
\text { s }\end{array}$} & \multicolumn{4}{|c|}{ Simulation modelling } & \multicolumn{4}{|c|}{ Math modelling } \\
\hline & $\overline{q_{i}}$ & $v_{q_{i}}$ & $\overline{h_{i}}$ & $\overline{q_{i}}$ & $v_{q}$ & $\overline{h_{i}}$ & $\overline{q_{i}}$ & $v_{q_{i}}$ \\
\hline 1 & 27.05 & 0.217 & 766 & 0.411 & 27.27 & 0.259 & 791.18 & 0.416 \\
\hline 2 & 36.86 & 0.215 & 755 & 0.403 & 26.64 & 0.265 & 759.15 & 0.499 \\
\hline 3 & 3.08 & 0.782 & 15.3 & 1.37 & 3.19 & 0.980 & 19.9 & 1.22 \\
\hline 4 & 15.29 & 0.258 & 249 & 0.484 & 15.12 & 0.262 & 244.4 & 0.498 \\
\hline 5 & 15.29 & 0.264 & 260 & 0.508 & $15 / 57$ & 0.256 & 258.3 & 0.487 \\
\hline $\begin{array}{c}\text { A } \\
\text { source } \\
\text { (Node } \\
0)\end{array}$ & $\begin{array}{c}\overline{\sum Q_{j}} \\
= \\
513,5 \\
6\end{array}$ & ${ }^{v_{\sum, 205} Q_{i}}=$ & $\begin{array}{c}\overline{H_{\Delta}}= \\
1,4\end{array}$ & $\begin{array}{l}v_{H_{\Delta}}= \\
0,410\end{array}$ & $\begin{array}{c}\bar{\sum} Q_{j} \\
513,1\end{array}$ & $\begin{array}{c}{ }^{U} \sum_{=} Q_{i} \\
0,189\end{array}$ & $\begin{array}{c}\overline{H_{\Delta}}= \\
1,35\end{array}$ & $\begin{array}{c}v_{H_{\Delta}} \\
= \\
0,408\end{array}$ \\
\hline
\end{tabular}

Comparison of simulation results and calculations of the mathematical model of stochastic flow distribution for the network in Figure 3 


\begin{tabular}{|c|c|c|c|c|c|c|c|c|}
\hline \multirow{2}{*}{$\begin{array}{l}\text { Rooms } \\
\text { plot } \\
\text { networks }\end{array}$} & \multicolumn{4}{|c|}{ Simulation modelling } & \multicolumn{4}{|c|}{ Math modelling } \\
\hline & $q_{i}$ & $v_{q u}$ & $\overline{h_{i}}$ & $V_{h_{u}}$ & $q_{i}$ & $v_{q u}$ & $\overline{h_{i}}$ & $V_{h_{u}}$ \\
\hline 1 & 2 & 3 & 4 & 5 & 6 & 7 & 8 & 9 \\
\hline 1 & 136.89 & 0.388 & 1.28 & 0.684 & 137.0 & 0.391 & 1.33 & 0.691 \\
\hline 2 & 376.67 & 0.396 & 2.13 & 0.703 & 376.1 & 0.391 & 2.01 & 0.701 \\
\hline 3 & 80.22 & 0.396 & 3.45 & 0.705 & 80 & 0.39 & 3.25 & 0.695 \\
\hline 4 & 50.95 & 0.381 & 1.72 & 0.679 & 51 & 0.389 & 1.8 & 0.683 \\
\hline 5 & 14.51 & 0.389 & 4.16 & 0.711 & 14.5 & 0.394 & 4.12 & 0.699 \\
\hline 6 & 30.74 & 0.385 & 2.34 & 0.707 & 30.8 & 0.388 & 2.41 & 0.715 \\
\hline 7 & 14.99 & 0.399 & 4.44 & 0.71 & 15.1 & 0.403 & 4.51 & 0.78 \\
\hline 8 & 169.23 & 0.397 & 2.9 & 0.689 & 161.3 & 0.399 & 2.95 & 0.702 \\
\hline 9 & 194.6 & 0.391 & 2.59 & 0.694 & 195.1 & 0.402 & 2.71 & 0.71 \\
\hline 10 & 159.8 & 0.393 & 2.79 & 0.695 & 159.1 & 0.389 & 2.67 & 0.691 \\
\hline 11 & 95.5 & 0.384 & 2.43 & 0.721 & 95.4 & 0.381 & 2.41 & 0.72 \\
\hline 12 & 15.07 & 0.401 & 2.63 & 0.711 & 15.3 & 0.411 & 2.72 & 0.719 \\
\hline 13 & 75.15 & 0.409 & 2.7 & 0.771 & 74.8 & 0.4 & 2.63 & 0.769 \\
\hline 14 & 14.39 & 0.448 & 3.64 & 0.745 & 14.5 & 0.451 & 3.72 & 0.75 \\
\hline 15 & 52.1 & 0.444 & 4.68 & 0.691 & 52.9 & 0.449 & 4.76 & 0.688 \\
\hline 16 & 90.41 & 0.388 & 2.49 & 0.699 & 90.1 & 0.381 & 2.41 & 0.698 \\
\hline 17 & 139.7 & 0.399 & 2.33 & 0.72 & 138.3 & 0.388 & 2.21 & 0.69 \\
\hline 18 & 75.67 & 0.428 & 3.14 & 0.714 & 75.2 & 0.417 & 3.1 & 0.702 \\
\hline 19 & 107.3 & 0.409 & 3.56 & 0.722 & 108.3 & 0.415 & 3.72 & 0.735 \\
\hline 20 & 86.41 & 0.419 & 4.04 & 0.807 & 86.2 & 0.403 & 3.91 & 0.798 \\
\hline 21 & 37.99 & 0.446 & 2.49 & 0.701 & 37.7 & 0.425 & 2.33 & 0.692 \\
\hline 22 & 54.15 & 0.399 & 1.54 & 0.734 & 54.01 & 0.391 & 1.52 & 0.733 \\
\hline 23 & 16.54 & 0.428 & 5.39 & 0.655 & 16.1 & 0.421 & 5.23 & 0.651 \\
\hline 24 & 3.31 & 0.345 & 0.94 & 0.755 & 3.44 & 0.355 & 0.99 & 0.761 \\
\hline 25 & 37.92 & 0.448 & 4.62 & 0.709 & 37.7 & 0.432 & 4.24 & 0.697 \\
\hline 26 & 18.1 & 0.399 & 3.24 & 0.727 & 18.2 & 0.405 & 3.41 & 0.731 \\
\hline 27 & 52.61 & 0.424 & 4.99 & 0.686 & 52.2 & 0.421 & 4.91 & 0.683 \\
\hline 28 & 21.33 & 0.37 & 1.07 & 0.726 & 20.6 & 0.362 & 1.08 & 0.701 \\
\hline 29 & 70.52 & 0.426 & 0.89 & 0.759 & 71 & 0.431 & 0.95 & 0.772 \\
\hline 30 & 10.01 & 0.441 & 6.34 & 0.726 & 9.97 & 0.417 & 0.14 & 0.711 \\
\hline 31 & 46.3 & 0.423 & 6.49 & 0.709 & 46.2 & 0.421 & 6.21 & 0.695 \\
\hline 32 & 16.02 & 0.405 & 3.63 & 0.751 & 16.41 & 0.396 & 3.47 & 0.742 \\
\hline 33 & 3.86 & 0.386 & 1.32 & 0.696 & 4.22 & 0.392 & 1.41 & 0.707 \\
\hline 34 & 14.87 & 0.371 & 3.06 & 0.898 & 14.33 & 0.361 & 3 & 0.876 \\
\hline 35 & 8.85 & 0.58 & 0.89 & 0.778 & 8.49 & 0.471 & 0.83 & 0.77 \\
\hline 36 & 48.79 & 0.449 & 0.95 & 0.717 & 49.2 & 0.457 & 0.99 & 0.731 \\
\hline 37 & 8.6 & 0.411 & 6.66 & 0.777 & 8.56 & 0.4 & 6.51 & 0.769 \\
\hline 38 & 32.67 & 0.471 & 3.29 & 3.29 & 0.775 & 32.4 & 0.469 & 0.768 \\
\hline 39 & 21.13 & 0.432 & 3.48 & 3.48 & 0.931 & 21 & 0.43 & 0.927 \\
\hline 40 & 4.9 & 0.632 & 0.402 & 0.807 & 4.88 & 0.622 & 0.389 & 0.8 \\
\hline 41 & 21.18 & 0.474 & 0.44 & 1.39 & 20.3 & 0.465 & 0.37 & 1.2 \\
\hline
\end{tabular}




\section{Continuation}

\begin{tabular}{|c|c|c|c|c|c|c|c|c|}
\hline \multirow{2}{*}{$\begin{array}{c}\text { Rooms } \\
\text { plot } \\
\text { networks }\end{array}$} & \multicolumn{4}{|c|}{ Simulation modelling } & \multicolumn{4}{|c|}{ Math modelling } \\
\cline { 2 - 9 } & $\overline{q_{i}}$ & $v_{q_{i}}$ & $\overline{h_{i}}$ & $v_{h_{u}}$ & $\overline{q_{i}}$ & $v_{q_{i}}$ & $\overline{h_{i}}$ & $v_{h_{u}}$ \\
\hline 42 & 2.75 & 0.791 & 0.83 & 0.792 & 2.92 & 0.81 & 0.98 & 0.82 \\
\hline 43 & 26.12 & 0.449 & 1.47 & 0.678 & 26.3 & 0.44 & 1.41 & 0.67 \\
\hline 44 & 18.39 & 0.382 & 3.77 & 0.754 & 18.61 & 0.389 & 0.98 & 0.781 \\
\hline 45 & 18.67 & 0.444 & 0.86 & 0.784 & 18.5 & 0.437 & 0.78 & 0.765 \\
\hline 46 & 7.56 & 0.429 & 3.52 & 0.769 & 7.69 & 0.435 & 8.68 & 0.782 \\
\hline 47 & 6.37 & 0.418 & 3.68 & 0.769 & 6.32 & 0.401 & 3.52 & 0.761 \\
\hline 48 & 7.28 & 0.426 & 0.13 & 0.836 & 7.15 & 0.397 & 0.12 & 0.811 \\
\hline 49 & 8.62 & 0.391 & 6.59 & 0.707 & 8.53 & 0.376 & 6.31 & 0.696 \\
\hline 50 & 2.16 & 0.822 & 5.48 & 1.71 & 2.03 & 0.802 & 5.31 & 1.63 \\
\hline A source & $\bar{\sum} Q_{j}=$ & $v_{\sum Q_{i}}=$ & $\overline{H_{\Delta}}$ & $v_{H_{\Delta}}=$ & $\bar{\sum} Q_{j}$ & $v_{\sum} Q_{i}$ & $\overline{H_{\Delta}}$ & $v_{H_{\Delta}}$ \\
(Node 0) & 513.56 & 0.205 & 1.4 & 0.410 & 513.1 & 0.189 & 1.35 & 0.408 \\
\hline
\end{tabular}

\section{Conclusions}

In this article, we propose ways to implement a quasilinear mathematical model of flow distribution in pipeline engineering networks, which determines the matrices of generalized network parameters - load distribution coefficients along the branches of the scheme, calculated at a point corresponding to the mathematical expectation of node loads. Based on the model obtained in the work, the convergence of the obtained results with the results of simulation modelling of engineering networks is proved by numerical experiment on an electronic computer.

The effectiveness of the developed model, the corresponding algorithms and the software package is proved. The values of the criterion of reduced costs for parametric optimization of engineering networks are given by comparing the results obtained, it is shown that they can be reduced in comparison with the methods currently used in practice. The article indicates the possibility of obtaining equivalent hydraulic characteristics of engineering networks at the design stage in the following cases.

\section{References}

1. Umarkhodja Baxramov. Development of a mathematical model of stochastic flow distribution in pipeline engineering networks, TASHIIZhT, Bulletin. Quarterly. 1. (2015)

2. Umarkhodja Baxramov. Determination of the stochastic parameters of flow distribution in engineering networks, TASHIIZhT, Bulletin Quarterly. 1. (2016)

3. Buslenko N.P., Golenko D.I., Sobol I.M., Sragovich V.G., Shreider Yu.A. Statistical Test Method (Monte Carlo Method) -M .: Fizmatgiz. -332 p. (1962).

4. Umarkhodja Baxramov, Irkin Makhamataliev. Modeling of the Random Process of Changing the Structure of an Engineering Network, International Jornal of Scientific \& Technology Research, 9 (2), pp. 2676-2678. (2020)

5. Umarkhodja Baxramov, G.K.Abduganieva. Determination of the reliability of pipeline engineering networks during their work in various conditions. Bulletin of Karakalpok State University named after Berdakh, 2 (34) 2017).

6. Chetverikov I.N., Belyakovich E.A., Menkov A.V. Computing technology for 
statistical modeling.-M, Sov.radio, -321 p. (1978).

7. Umarkhodja Baxramov, G.K. Abdiganieva. Methods for describing the loads of a water supply and distribution system for long-term forecasting. "Bulletin of Karakalpak State University named after Berdakh .Nukus.2019. No. 1 (38) 2018, ISSN 2010-9075

8. Halperin EM. Reliability of water supply and sanitation systems, Modern problems of science and education, 1. 2009

9. Lykin A.V. Mathematical modeling of electrical systems and their elements, 2nd edition, Novosibirsk: Publishing house NSTU.-228 p. (2009)

10. Sveshnikov A.A. Applied methods of the theory of random functions, M., Nauka 232 p. (1968) 